\title{
Arranjo de subestação: um estudo de revisão bibliográfica
}

\author{
Substation arrangement: a literature review study \\ Disposición de la subestación: un estudio de revisión de la literatura
}

Recebido: 14/10/2021 | Revisado: 24/10/2021 | Aceito: 31/10/2021 | Publicado: 01/11/2021

\author{
Paulo Roberto Duailibe Monteiro \\ ORCID: https://orcid.org/0000-0002-7376-9115 \\ Universidade Federal Fluminense, Brasil \\ E-mail: pauloduailibe@id.uff.br \\ Jullia Mercedes Miranda \\ ORCID: https://orcid.org/0000-0001-8949-282X \\ Universidade Federal Fluminense, Brasil \\ E-mail: julliamercedes@id.uff.br
}

\begin{abstract}
Resumo
A energia elétrica desempenha um papel socioeconômico fundamental no mundo. No que tange a esse contexto, as subestações são de grande relevância uma vez que são usadas para dirigir o fluxo de energia em um sistema de potência e possibilitar a sua diversificação através de rotas alternativas e também permitem a distribuição de energia elétrica para atendimento aos consumidores residenciais, comerciais e industriais no adequado nível para utilização. A crescente demanda por eletricidade e consequentemente a expansão do sistema elétrico tem colaborado para o aumento de estudos na área. Este trabalho apresenta um estudo bibliográfico, a partir de uma análise bibliométrica, das pesquisas desenvolvidas na área de arranjo de subestações. A revisão bibliográfica é uma importante ferramenta para levantar a produção científica sobre o tema. Foram considerados para esse estudo produções acadêmicas de cursos de graduação, pós-graduação, artigos de periódicos e publicações em congressos nas plataformas Scopus e Periódicos Capes pela sua abrangência, organização dos dados e relevância. Os resultados da pesquisa indicam o que há de mais atual e as principais pesquisas na área de arranjo de subestações. No que tange aos países que mais publicaram nos últimos 5 anos sobre arranjo de subestações, destaque para a China que está em primeiro lugar com 14 publicações. Já o Brasil ocupa a sexta colocação em produções relacionadas sobre o tema.
\end{abstract}

Palavras-chave: Energia elétrica; Transmissão de energia; Subestações elétricas; Arranjo de subestação; Revisão bibliográfica.

\begin{abstract}
Electric energy plays a fundamental socio-economic role in the world. In this context, substations are of great importance since they are used to direct the flow of energy in a power system and enable its diversification through alternative routes and also allow the distribution of electricity to serve residential consumers, commercial and industrial at the appropriate level for use. The growing demand for electricity and consequently the expansion of the electrical system has contributed to the increase in studies in the area. This work presents a bibliographical study, from a bibliometric analysis, of the researches developed in the area of substation arrangement. Literature review is an important tool to raise scientific production on the subject. For this study, academic productions from undergraduate and graduate courses, journal articles and publications in conferences on the Scopus and Capes Periodicals platforms were considered due to their scope, organization of data and relevance. The research results indicate the most current and the main researches in the area of substation arrangement. Regarding the countries that have published the most in the last 5 years about the arrangement of substations, China stands out, which is in first place with 14 publications. Brazil occupies the sixth position in related productions on the subject.
\end{abstract}

Keywords: Electric power; Power transmission; Electrical substations; Substation arrangement; Literature review.

\section{Resumen}

La energía eléctrica juega un papel socioeconómico fundamental en el mundo. En este contexto, las subestaciones son de gran importancia ya que se utilizan para dirigir el flujo de energía en un sistema eléctrico y permitir su diversificación a través de rutas alternativas y también permiten la distribución de electricidad para servir a los consumidores residenciales, comerciales e industriales en el nivel adecuado para usar. La creciente demanda de energía eléctrica y consecuentemente la expansión del sistema eléctrico ha contribuido al incremento de estudios en la zona. Este trabajo presenta un estudio bibliográfico, a partir de un análisis bibliométrico, de las investigaciones desarrolladas en el área de disposición de subestaciones. La revisión de la literatura es una herramienta importante para elevar la producción científica sobre el tema. Para este estudio se consideraron producciones académicas de pre y posgrado, artículos de revistas y publicaciones en congresos en las plataformas Scopus y Capes Periodicals por su 
alcance, organización de datos y relevancia. Los resultados de la investigación indican las investigaciones más actuales y principales en el área de disposición de subestaciones. En cuanto a los países que más han publicado en los últimos 5 años sobre la disposición de subestaciones, destaca China, que ocupa el primer lugar con 14 publicaciones. Brasil ocupa la sexta posición en producciones relacionadas sobre el tema.

Palabras clave: Energía eléctrica; Transmisión de potencia; Subestaciones eléctricas; Disposición de subestaciones; Revisión de la literatura.

\section{Introdução}

As usinas responsáveis por gerar energia elétrica para abastecer residências, indústrias, geralmente, ficam distantes do ponto de consumo final. Assim, a rede de transmissão de energia elétrica e suas subestações (SEs) assumem relevante importância para realizar o transporte da energia gerada num ambiente onde são exisgidos altos de níveis desempenho e segurança. Nesse sentido, o arranjo da SE exerce importância significativa no planejamento, pois é essencial para o bom desempenho do sistema elétrico e para elevar sua performance, transportando assim, a energia da forma mais eficiente possível (Marson, 2017)

A definição do arranjo das SEs é uma parte importante em um projeto, uma vez que possui alto impacto nos custos do projeto e também no resultado esperado do mesmo. Marson (2017) destaca a importância de investimentos direcionados ao sistema elétrico e acrescenta que os Sistemas Elétricos de Potência (SEP) podem contribuir para utilização da energia de forma eficiente e sustentável, colaborando, inclusive, para o crescimento da indústria. Além disso, alerta sobre a configuração utilizada no Brasil uma vez que os sistemas de energia elétrica demonstram que diversas melhorias e investimentos ainda se fazem necessários.

Estudo de arranjo de SEs é um assunto bastante complexo, devido a vários fatores que devem ser considerados na análise e ao carater da abordagem que as vezes possuem aspectos contraditório, como por exemplo, arranjos que apresentam simplicidade operacional e do sistema de proteção, o que é desejável, sem, no entanto, apresentar outras características importantes para a operação do sistema. Diversos fatores influenciam na definição do esquema de barramento de SE mais adequado, tais como o custo, a facilidade do arranjo físico, a confiabilidade, a flexibilidade operacional, a facilidade de expansão, a segurança, e o sistema de proteção e controle. Devido à importância e sua complexidade de análise, o arranjo de SEs tem sido objeto de estudo de diversos autores e instituições.

Souza (2003) apresenta a metodologia e modelos de simulação para comparação de arranjos de subestações de distribuição através de estudo de confiabilidade estática de esquemas de manobra. Na análise o autor realizou a avaliação de confiabilidade utilizando dois índices, a saber: LOLP (Loss of Load Probability) que se refere à soma de todas as probabilidades de possíveis falhas; e LOLE (Loss of Load Expectation) que se refere à soma das expectativas de condições de falha. Brandão (2008) aborda também o cálculo da confiabilidade de subestações utilizando o método de Markov a fim de verificar a influência da sobrecarga na confiabilidade de uma subestação.

Um estudo comparativo de arranjos de subestação com sua respectiva análise de performance e também de economicidade é realizado por Ribeiro et al (2011). A metodologia do estudo baseia-se na análise do fluxo de potência, o cálculo do curto-circuito, a confiabilidade e a análise econômica. O planejamento e análise do arranjo de subestação são etapas importantes em um projeto de sistema elétrico uma vez que essa definição impacta diretamente na qualidade da energia do sistema, conforme Ruback e Costa (2018).

Colussi (2002) conclui em seu estudo que existe uma tendência das empresas de transmissão de energia elétrica adotarem procedimentos padrões na definição do tipo de arranjo de subestações. Dito isto, o tipo de arranjo a ser utilizado é definido de acordo com os níveis de tensão da subestação. Essa tendência à padronização é reforçada pelo Operador Nacional do Sistema Elétrico (ONS) que definiu o arranjo das subestações de acordo com o nível de tensão de operação.

Segundo Conceição et al (2016) em um estudo sobre transmissão de energia elétrica em ultra alta tensão, a utilização 
de ultra alta tensão em rede de transmissão é uma tecnologia que está em desenvolvimento na China e na Rússia e chega ao Brasil com a construção da Usina Hidrelétrica (UHE) de Belo Monte, objeto de estudo da pesquisa, localizada na bacia do Rio Xingu, próximo ao município de Altamira, no norte do estado Pará. A usina é constituída de três subsistemas de acordo com o nível de tensão, de 500, 230 e 69 kV. As estações conversoras são responsáveis pelo processo de conversão de $500 \mathrm{kV}$ em corrente alternada para $800 \mathrm{kV}$ em corrente continua possibilitando o transporte de energia nessa tensão para o estado do Rio de Janeiro e Minas Gerais, sendo novamente convertido para $500 \mathrm{kV}$ em corrente alternada para a interação com o sistema interligado nacional.

De acordo com um estudo de Silveira (2017) um sistema de transmissão em ultra alta tensão (UAT) em corrente alternada em um nível de tensão de $1000 \mathrm{kV}$, ainda não utilizado no Brasil, mas já utilizado no mundo para transmissão em grandes blocos de energia por longas distâncias em países como China, Índia, Japão e Rússia. Novos estudos de arranjos de SE para esse nível de tensão serão necessários.

Conforme a EPE (2020), a demanda por energia elétrica aumentou consideravelmente nos últimos anos através de políticas públicas e devido, principalmente, ao crescimento da economia nacional. Esse aumento do consumo cria a necessidade de adaptação e expansão do setor de energia, desde o processo de geração, transmissão até a distribuição de energia elétrica. Além disso, uma grande parcela da energia consumida no Brasil decorre de indústrias. Conceição et al (2016) conclui que com a crescente demanda de energia elétrica é ainda mais relevante o estudo sobre novas tecnologias para transmissão em grandes blocos de energia, já de acordo com Leite et al. (2019), é necessária a implementação de medidas que possibilitem não só o aumento da oferta de energia por meio de fontes renováveis, mas também o seu uso de maneira racional e eficiente. Com a permanente expansão do setor elétrico e as necessidades pela preservação do meio ambiente ambientais, impondo energias mais limpas e renováveis, é esperado um aumento na demanda por projetos de modernas SEs com a utilização de novas tecnologias e inovações nos arranjos. A Figura 1 apresenta um exemplo de sistema elétrico simplificado.

Figura 1 - Sistema Elétrico Simplificado.

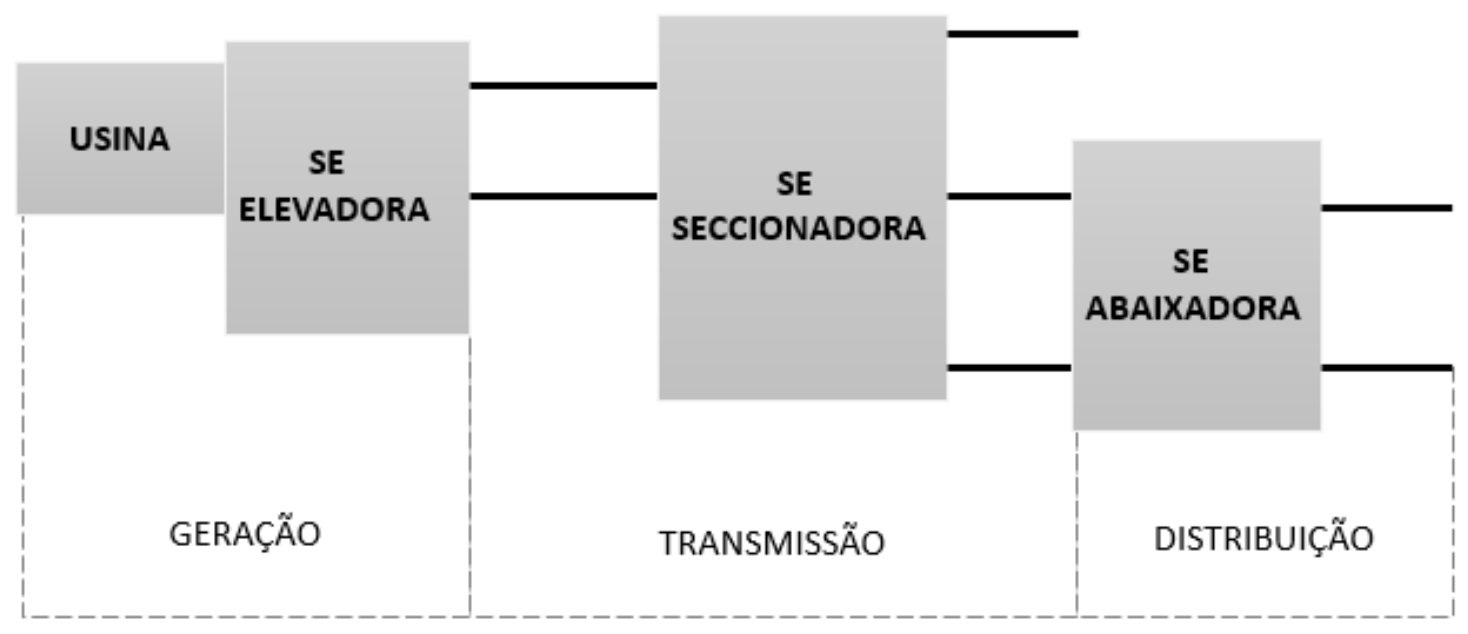

Fonte: Autores (2021).

Segundo Mamede Filho $(2013,2019)$ o barramento de uma subestação é um elemento essencial no sistema, pois concentra uma grande quantidade de potência e muitas derivações para atendimento de cargas elétricas, tornando um importante elemento para a garantia da confiabilidade do sistema. Além disso, o tipo de arranjo influencia na obtenção de uma maior ou menor flexibilidade operacional, o que impacta diretamente no desempenho da subestação. Os autores acrescentam ainda que arranjos mais complexos e com mais recursos operacionais aumentam, consequentemente, o investimento no sistema 
e também o nível de confiabilidade no mesmo. Arranjos mais simples demandam um investimento menor e em geral são utilizados em sistemas que não precisam de um alto nível de confiabilidade e continuidade.

Como exposto, o arranjo de SEs é de fundamental importância para o desempenho e segurança do sistema elétrico. Diante disso, este artigo se propôs a realizar uma revisão bibliográfica a partir de uma análise bibliométrica da produção científica sobre arranjo de SEs. Para tanto, foram utilizados artigos indexados na base de dados nas plataformas Scopus e Periódicos CAPES (Coordenação de Aperfeiçoamento de Pessoal de Nível Superior).

\section{Metodologia}

Segundo Cabeza et al 2020, a bibliometria consiste em uma das principais ferramentas de pesquisa que utiliza métodos estatísticos para analisar a produção acadêmica presente nos mais diversos tipos de documentos. A sua utilização acaba por medir quantitativamente a evolução da produção de conhecimento científico e tecnológico de um tema específico.

Segundo Ferreira (2002), as pesquisas denominadas como "estado da Arte" ou "estado do conhecimento" são fundamentadas em uma análise bibliográfica com o mapeamento das produções acadêmicas relacionadas ao objeto da pesquisa em diversos campos de atuação, aspectos e dimensões. Outrossim, também são utilizadas para um levantamento quantitativo e descritivo das produções acadêmicas da área da pesquisa.

Para realização da análise bibliométrica deste estudo, utilizou-se as bases de dados dos Periódicos CAPES (Coordenação de Aperfeiçoamento de Pessoal de Nível Superior) e Scopus com a finalidade de avaliar quantitativamente a produção científica sobre o tema de Arranjo de Subestações. As plataformas foram escolhidas pela abrangência e relevância no ambiente acadêmico.

\section{Resultados}

Subestação (SE) é um conjunto de equipamentos de manobra e/ou transformação e compensação usado para dirigir o fluxo de energia em um sistema de potência e possibilitar a sua diversificação através de rotas alternativas, possuindo dispositivos de proteção capazes de detectar falhas e isolar trechos. Elas podem ser classificadas quanto a sua função e instalação. Quanto a sua função podem ser subestações geradoras, transformadoras e seccionadora ou de manobra. Quanto a sua instalação elas podem ser ao tempo ou abrigadas.

$\mathrm{O}$ arranjo de barramento adotado para a SE determina o arranjo elétrico e físico dos equipamentos de manobra. Diversos fatores devem ser considerados para a definição do arranjo de uma SE tais como, simplicidade, economia, confiabilidade e segurança, conforme requerido pela função e importância da SE a ser projetada.

Os arranjos de barramento de SEs normalmente utilizados são: Barramento simples; barramento principal e de transferência; barramento duplo com disjuntor simples; barramento em anel; barramento disjuntor e meio e barramento duplo com duplo disjuntores. Em sistemas de transmissão o submódulo 2.3 do ONS (2020) estabelece as condições para conexão à rede básica, dos arranjos de barramentos para SEs com isolamento a ar (AIS - Air Insulated Switchgear) diferenciados por classe de tensão conforme descrito a seguir:

- Barramentos de $230 \mathrm{kV}$ : barra dupla com disjuntor simples a quatro chaves;

- Barramentos de tensão igual ou superior a $345 \mathrm{kV}$ : barra dupla com disjuntor e meio.

Nos sistemas de distribuição, normalmente a faixa de tensão vai de $13,8 \mathrm{kV}$ até $138 \mathrm{kV}$, os arranjos de SE normalmente utilizados são de barramento simples, barramento simples com recurso, barramento principal e de transferência e em alguns casos barramento duplo com disjuntor simples e barramento em anel.

Nos sistemas de distribuição industriais, exceto a SE de entrada, que depende do nível de tensão a que está conectada, os arranjos comumente usados são o radial; o primário seletivo; e o secundário seletivo ou combinações deles (Beeman, 1955). 
O primário seletivo provê alternativa contra perda de uma fonte de suprimento primária. Dois circuitos de distribuição primária alimentados por fontes distintas, ou barramentos distintos, alimentam as várias subestações situadas nos centros de carga. Cada subestação ou transformador é conectado a dois alimentadores primários independentes, via dispositivos de chaveamento, de modo a se obter uma fonte normal e uma fonte alternativa independentes.

Já, por sua vez, o secundário seletivo é o arranjo em que os dois barramentos do secundário dos transformadores estão conectados por um disjuntor de interligação. No caso de falha do circuito primário ou do transformador, o suprimento para as cargas da subestação pode ser mantido pelo disjuntor de interligação. O disjuntor de interligação pode operar normalmente aberto (NA) ou normalmente fechado (NF). A escolha da operação NA ou NF afeta o nível de curto-circuito do painel. Pode-se prever também, um sistema de transferência automática.

Tanto nos sistemas de transmissão, distribuição e industrial, grande parte das SEs construídas são com isolamento a ar devido aos baixos custos de investimento, entretanto outras tecnologias se apresentam como alternativas para soluções em determinadas situações. Assim, algumas empresas passaram a investir na construção de SEs isoladas a gás SF6 (GIS - gas insulated substations), que, apesar do custo elevado, têm excelentes propriedades de isolação e possuem equipamentos de tamanho reduzido de acordo com de Oliveira (2017). As GIS são soluções competitivas para áreas congestionadas ou com alto valor do terreno, para áreas com contaminantes no ar e regiões litorâneas.

As GIS são instalações compactas e apresentam como vantagens a alta confiabilidade, os longos intervalos entre manutenções, o baixo impacto visual, baixo custo do terreno/prédios e o baixo custo de montagem. Como desvantagens podese destacar o alto investimento inicial, o alto custo de reposição de componentes e a manutenção e operação especializadas. Assim, as GIS se apresentam como alternativas às SEs convencionais com isolamento a ar.

Segundo Heldt (2016), os arranjos de SE podem ter complexidade variadas, contudo a escolha do tipo de esquema afeta diretamente o desempenho da subestação. Os barramentos simples não são normalmente utilizados em SEs de alta relevância, pois trata-se de um esquema de barramento inflexível uma vez que há dependência de um único barramento principal, deixando o sistema suscetível a desligamentos frequentes. Nesse caso, em caso de falha ou necessidade de executar alguma manutenção na SE, obrigatoriamente, é preciso desligá-la por completo. A vantagem desse tipo de barramento é o baixo custo.

Já o barramento principal e transferência, segundo Colussi (2002), é um esquema em que se acrescenta um barramento de transferência ao esquema de barramento simples por meio de um disjuntor de amarre que liga os dois barramentos. Nesse caso quando um disjuntor é retirado para manutenção, o disjuntor de amarre mantém o circuito energizado. Contudo, se o barramento principal é retirado para manutenção nenhum disjuntor fica disponível para proteção do circuito. Ademais, o autor acrescenta que se houver falha ou necessidade de manutenção na barra principal ou em uma das chaves seccionadoras ligadas a mesa, ocorre a perda de todos os circuitas da subestação. Embora esse tipo de barramento não apresente um alto nível de flexibilidade e confiabilidade, este é um esquema muito utilizado no Brasil devido ao seu custo ser mais baixo em relação a outros tipos de esquemas.

$\mathrm{O}$ arranjo barramento duplo com disjuntor simples possui dois barramentos principais interligados por um disjuntor de amarre que, quando fechado, permite a transferência de um circuito de um barramento principal para outro, sem a necessidade de desenergizar o circuito. Esse processo de transferência é feito por meio das chaves seletoras de barramento de cada vão de entrada de circuito. Todos os circuitos podem operar ligados a um barramento principal ou ao outro, mas também pode operar com metade dos circuitos ligados a cada barramento. No primeiro caso há a perda de toda a SE em caso de falha em u disjuntor ou no barramento onde estão conectados todos os circuitos. No segundo caso, somente metade dos circuitos são desligados para o mesmo tipo de falha.

O barramento em anel é um esquema em que tem-se o mesmo número de disjuntores que de circuitos. Durante a 
operação normal todos os disjuntores permanecem fechados formando um anel. Em caso de falha em um circuito, dois disjuntores são desligados e, caso um dos disjuntores não abra, um circuito adicional é desligado pela operação da proteção de falha do disjuntor. De acordo com Giles (1970) e Anderson (1999) a vantagem do esquema com barramento em anel é que ele requer o uso de apenas um disjuntor por circuito, o que o toma econômico em custo e cada circuito de saída tem dois caminhos de alimentação, o que o torna mais flexível e confiável.

No caso de alimentação de várias SEs unitárias a partir de um sistema primário em anel, cada SE é conectada em série no anel alimentador primário via dispositivos de chaveamento, possibilitando uma alimentação dupla das SEs. Nesse tipo de sistema é necessária uma instrução eficiente da operação uma vez que a potência flui em duas direções. Neste tipo de sistema o anel pode operar aberto ou fechado.

O arranjo de barramento disjuntor e meio, é formado por três disjuntores em série entre dois barramentos principais. Os dois circuitos são ligados entre os barramentos principais, motivo pelo qual é chamado de disjuntor e meio. Neste tipo de esquema, em condições normais de operação, todos os disjuntores estão fechados e ambos barramentos energizados. Um circuito é desligado com a abertura dos dois disjuntores associados. Contudo, se houver falha do disjuntor central, um circuito adicional é desligado. Ademais, nenhum circuito adicional é desligado em caso de falha de um disjuntor ligado a um dos barramentos. Esse esquema é um dos mais caros, porém tem maior confiabilidade e flexibilidade uma vez que qualquer um dos barramentos pode ser retirado de operação sem a perda de nenhum circuito.

O barramento duplo com duplo disjuntor é composto por dois disjuntores para cada circuito. A operação pode ser feita com cada circuito ligado a ambos barramentos ou, parcialmente, com metade dos circuitos operando em cada um dos barramentos. Este tipo de esquema possui o preço elevado em relação a outros tipos de arranjo de barramentos porém, apresenta alto nível de confiabilidade quando a operação ocorre com todos os circuitos conectados em ambos barramentos.

Colussi (2002) aborda também em seu estudo os arranjos híbridos, que são combinações de diferentes tipos de arranjos, seja por superposição de sistemas ou utilização de arranjos com circuitos individuais. Esse tipo de arranjo é, geralmente, de alto custo e, ademais, complicados de operar devido às diversas formas de operação, ainda assim, o autor afirma que esse tipo de arranjo pode ser encontrado no Brasil em diversas subestações em operações de transmissão.

\section{Discussão}

Em busca realizada na plataforma CAPES, em agosto de 2021, observou-se que os assuntos alta, ultra e extra tensão vem sendo estudados, assim como o assunto sistema de distribuição de energia. No entanto, quando o assunto envolve diretamente subestações, a pesquisa passou a encontrar menos artigos, documentos e outros tipos de recurso. A seguir, o detalhamento da pesquisa realizada utilizando as seguintes palavras chaves no campo "assunto": subestação de alta tensão, subestações industriais, ultra alta tensão, extra alta tensão, alta tensão e sistemas de distribuição de energia. Como as pesquisas iniciais que utilizaram a palavra "subestação" no periódico CAPES, encontraram um baixo número de estudos, optou-se por não incluir nenhum filtro para as pesquisas realizada nesta plataforma.

Na Figura 2 é apresentado o volume de produções acadêmicas de acordo com a palavra chave utilizada na busca na plataforma periódico Capes. 
Figura 2 - Número de documento do periódicos Capes.

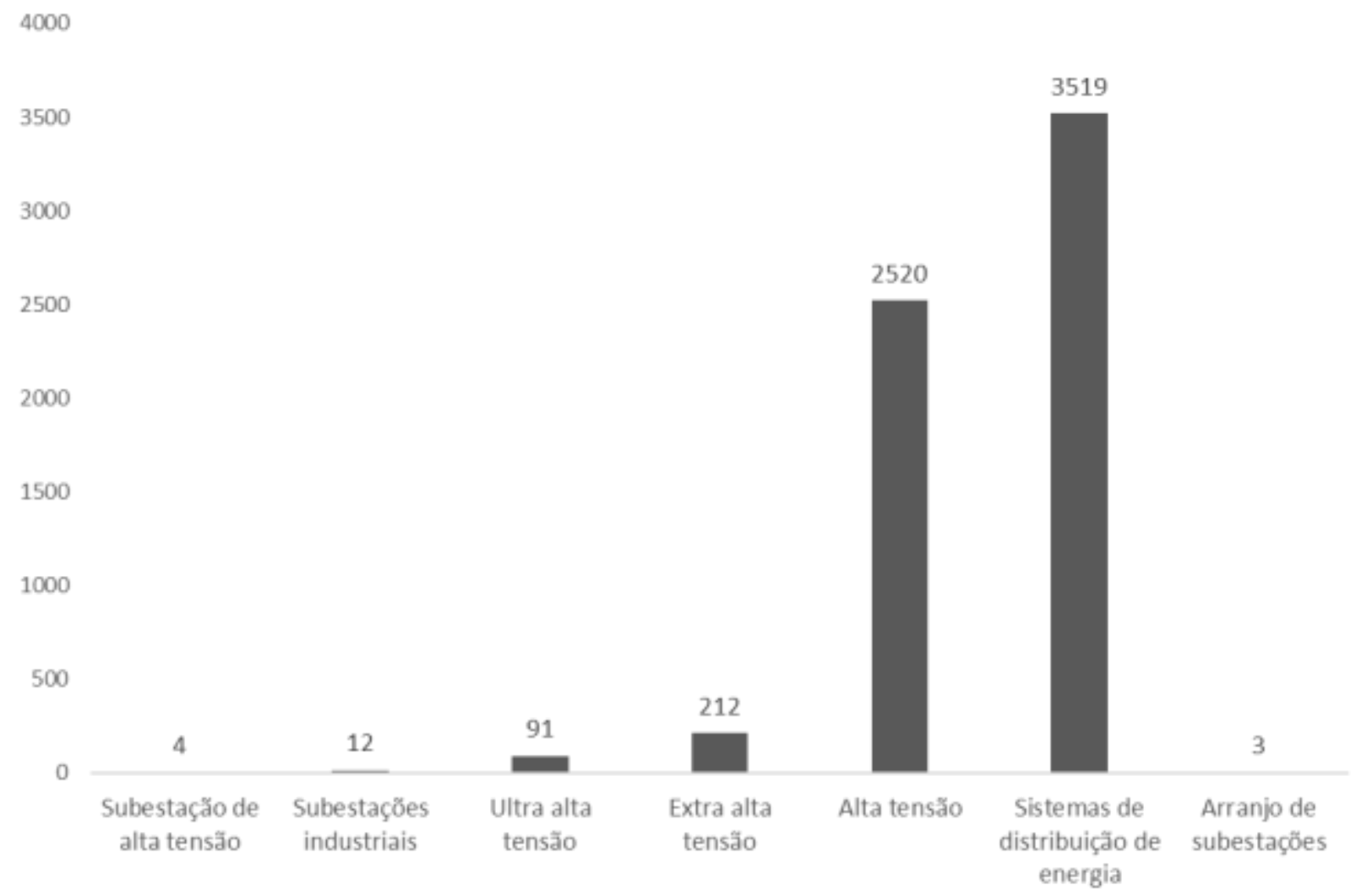

Fonte: Autores (2021).

Como pode ser observado na Figura 1 os termos que mais retornaram pesquisas na área foram "Sistemas de distribuição de energia" e "Alta tensão". O volume de produções acadêmicas relacionadas a estes termos é muito superior que as demais palavras chaves utilizadas na busca.

$\mathrm{Na}$ Tabela 1 é apresentado um detalhamento das produções acadêmicas de acordo com o tipo de documento elaborado.

Tabela 1 - Busca realizada no periódico Capes.

\begin{tabular}{|c|c|c|c|c|c|c|c|}
\hline $\begin{array}{l}\text { Palavra chave utilizada para busca na } \\
\text { plataforma Periódicos Capes. }\end{array}$ & Artigos & Livro & Tese & $\begin{array}{l}\text { Recurso } \\
\text { textual }\end{array}$ & Resenhas & $\begin{array}{l}\text { Atas de } \\
\text { Congresso }\end{array}$ & Total \\
\hline Subestação de alta tensão & 3 & 0 & 0 & 1 & 0 & 0 & 4 \\
\hline Subestações industriais & 8 & 2 & 1 & 1 & 0 & 0 & 12 \\
\hline Ultra alta tensão & 59 & 31 & 0 & 0 & 1 & 0 & 91 \\
\hline Extra alta tensão & 158 & 53 & 0 & 1 & 0 & 0 & 212 \\
\hline Alta tensão & 2217 & 250 & 25 & 20 & 7 & 1 & 2520 \\
\hline Sistemas de distribuição de energia & 3245 & 245 & 7 & 21 & 1 & 0 & 3519 \\
\hline Arranjo de subestações & 3 & 0 & 0 & 0 & 0 & 0 & 3 \\
\hline Total de publicações: & 5693 & 581 & 33 & 44 & 9 & 1 & 6361 \\
\hline
\end{tabular}

Fonte: Autores (2021).

De acordo com o levantamento realizado na Tabela 1 é possível verificar que o maior volume de produções na área, considerando as palavras chaves descritas na tabela, é de artigos conforme estudo realizado pela plataforma Capes.

Com o intuito de realizar uma pesquisa mais direcionada e atual, na plataforma Scopus, optou-se por utilizar o filtro 
para as pesquisas realizadas nos últimos 5 anos (2017 a 2021). Como a base de dados apresentou mais resultados quando a busca foi realizada em inglês, optou-se por realizar as seguintes palavras chave: high voltage substation, industrial substations, ultra high voltage, extra high voltage, high voltage, power distribution systems e substation arrangements. Como nas primeiras buscas encontrou-se uma quantidade de estudos significativa que não estava relacionada diretamente a área de estudos da energia, fez-se necessária a aplicação do filtro "energy" no campo "subject area". Abaixo, o quantitativo encontrado de documentos que utilização as palavras descritas anteriormente em seu título, resumo ou palavra chave.

Tabela 2 - Busca realizada no periódico Scopus.

\begin{tabular}{|c|c|c|c|c|c|c|c|c|}
\hline $\begin{array}{l}\text { Palavra chave utilizada para busca na } \\
\text { plataforma Scopus. }\end{array}$ & $\begin{array}{l}\text { Artigo de } \\
\text { Congresso }\end{array}$ & Artigo & $\begin{array}{l}\text { Capítulo } \\
\text { de Livro }\end{array}$ & $\begin{array}{c}\text { Atas de } \\
\text { Congresso }\end{array}$ & Anais & Livro & $\begin{array}{c}\text { Artigos de } \\
\text { Negócio }\end{array}$ & Total \\
\hline Subestação de alta tensão & 439 & 351 & 6 & 7 & 7 & 1 & 1 & 812 \\
\hline Subestações industriais & 137 & 54 & 3 & 7 & 2 & 0 & 0 & 203 \\
\hline Ultra alta tensão & 25 & 39 & 0 & 1 & 0 & 0 & 0 & 65 \\
\hline Extra alta tensão & 16 & 15 & 0 & 0 & 1 & 0 & 0 & 32 \\
\hline Alta tensão & 15776 & 18867 & 159 & 165 & 550 & 5 & 10 & 35532 \\
\hline Sistemas de distribuição de energia & 11319 & 11415 & 180 & 213 & 464 & 16 & 4 & 23611 \\
\hline Arranjo de subestações & 27 & 28 & 0 & 1 & 0 & 0 & 0 & 56 \\
\hline Total de publicações: & 27739 & 30769 & 348 & 394 & 1024 & 22 & 15 & 60311 \\
\hline
\end{tabular}

Fonte: Autores (2021).

A Figura 3 apresenta os autores que mais publicaram no assunto "high voltage substation" nos últimos 5 anos:

Figura 2 - Autores que mais produziram no tema "high voltage substation" na Scopus.

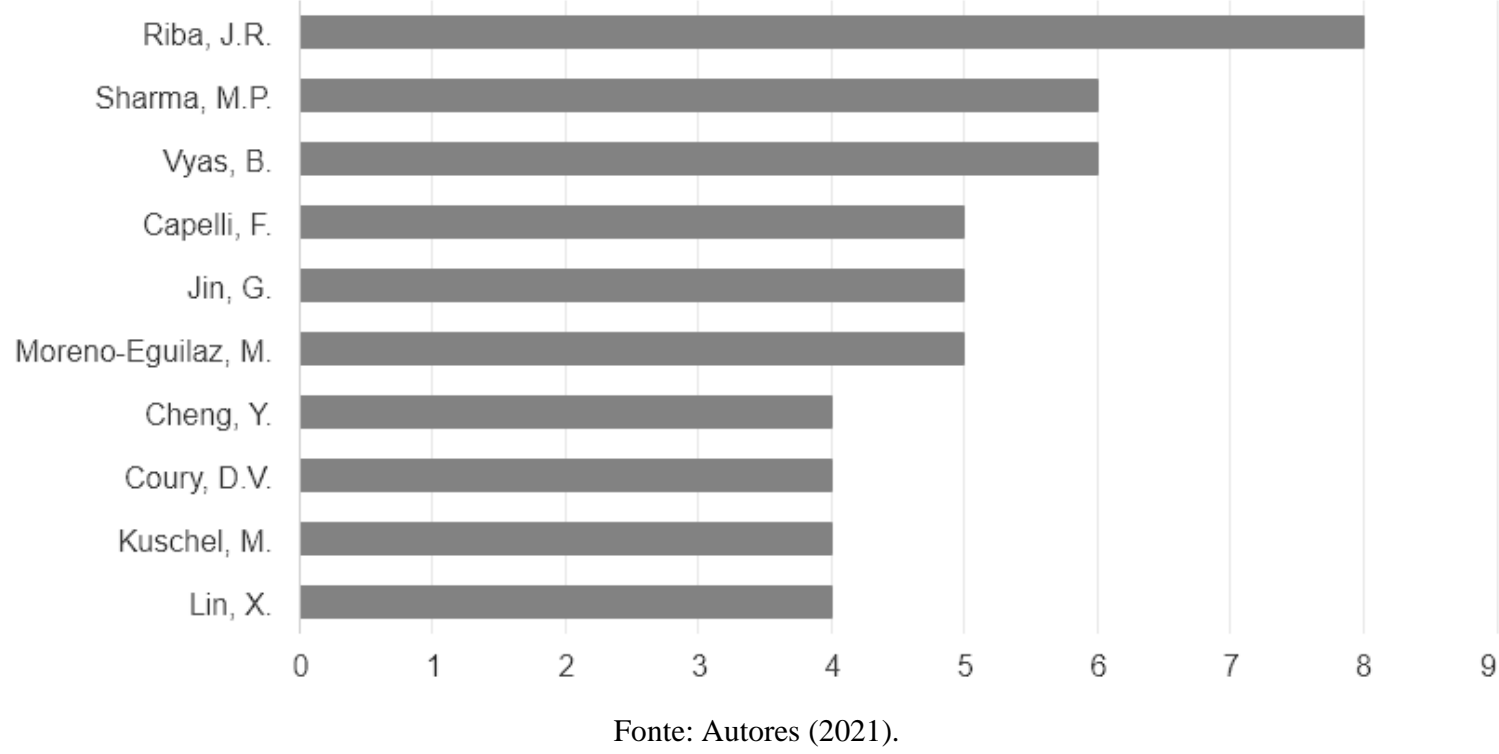

Como pode ser visto na Figura 3 o autor que mais publicou sobre o tema de subestações de alta tensão foi o JordiRoger Riba Ruiz, da Universidade Politécnica da Catalunha (Espanha). Nos últimos 5 anos foram 8 publicações relacionadas ao tema, além disso, foi citado por inúmeros autores.

E a seguir, na Figura 4, mostra-se as instituições (afiliações) que mais publicaram documentos no assunto "high voltage substation" nos últimos 5 anos. 
Research, Society and Development, v. 10, n. 14, e287101421805, 2021

(CC BY 4.0) | ISSN 2525-3409 | DOI: http://dx.doi.org/10.33448/rsd-v10i14.21805

Figura 3 - Afiliações que mais produziram no tema "high voltage substation" na Scopus.

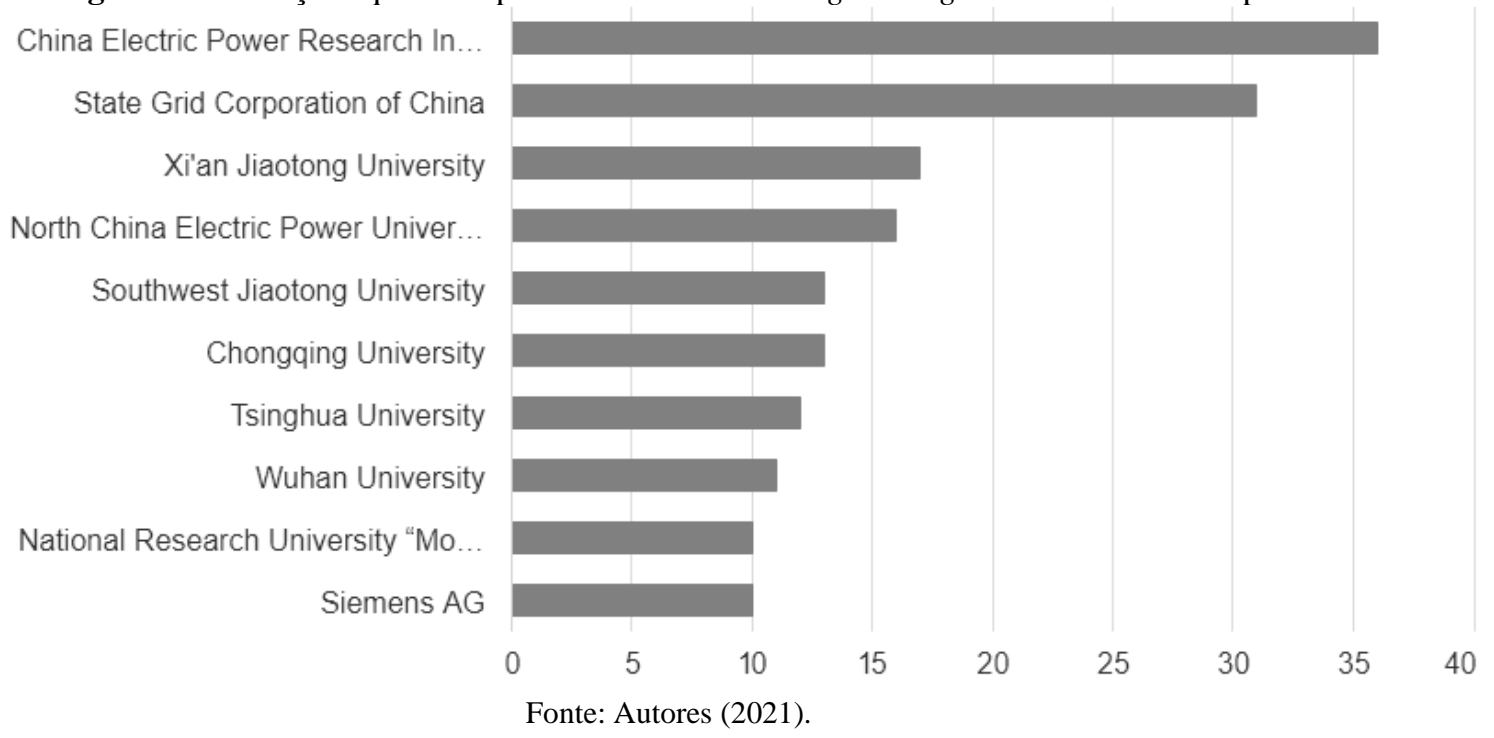

Na Figura 4 são relacionadas as principais instituições com produções acadêmicas no que tange ao assunto “subestações de alta tensão". Como pode ser visto, as afiliações da China foram as que mais publicaram estudo relacionado ao tema, conforme já citado ao longo desse artigo e ratificado pela análise bibliográfica.

Quanto ao número de documentos publicados nos últimos 5 anos no assunto "power distribution systems", a Figura 5 mostra a linha do tempo de produções relacionadas a essas palavras chaves.

Figura 4 - Quantidade de documentos publicados no tema "power distribution systems" na Scopus.

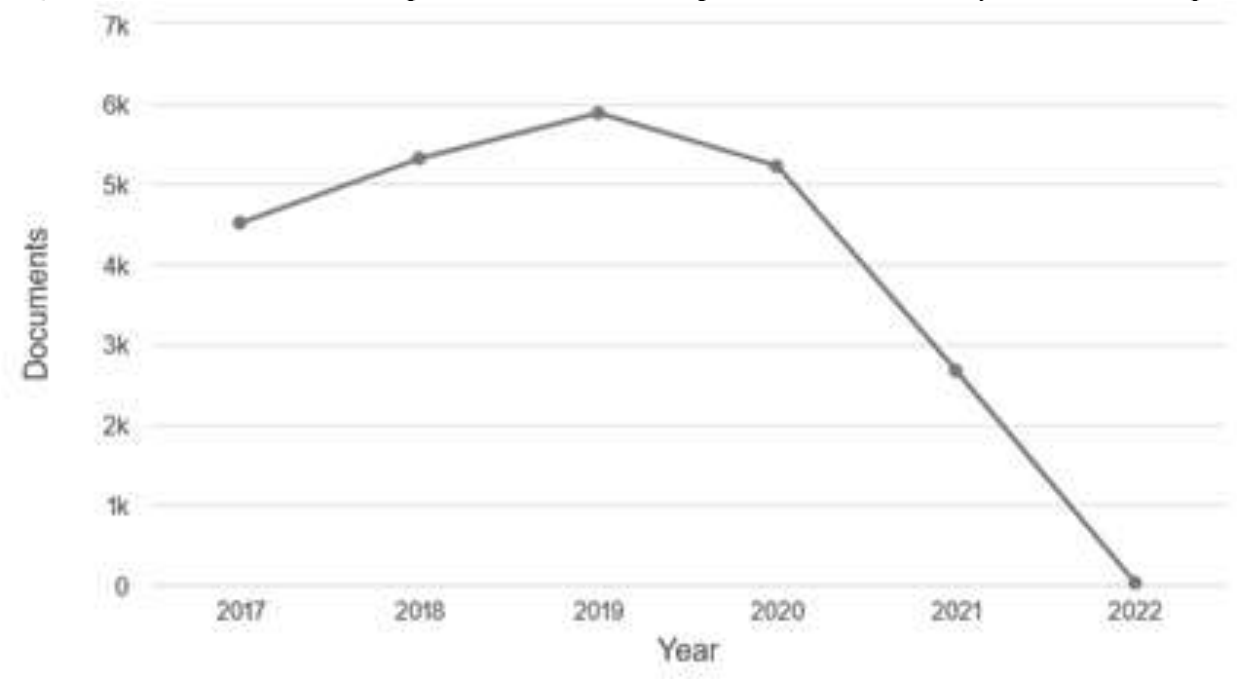

Fonte: Autores (2021).

No tema "arranjo de subestações", a pesquisa no Scopus encontrou 56 documentos. Abaixo os autores que mais publicaram no assunto "substation arrangements" nos últimos 5 anos conforme Figura 6. 


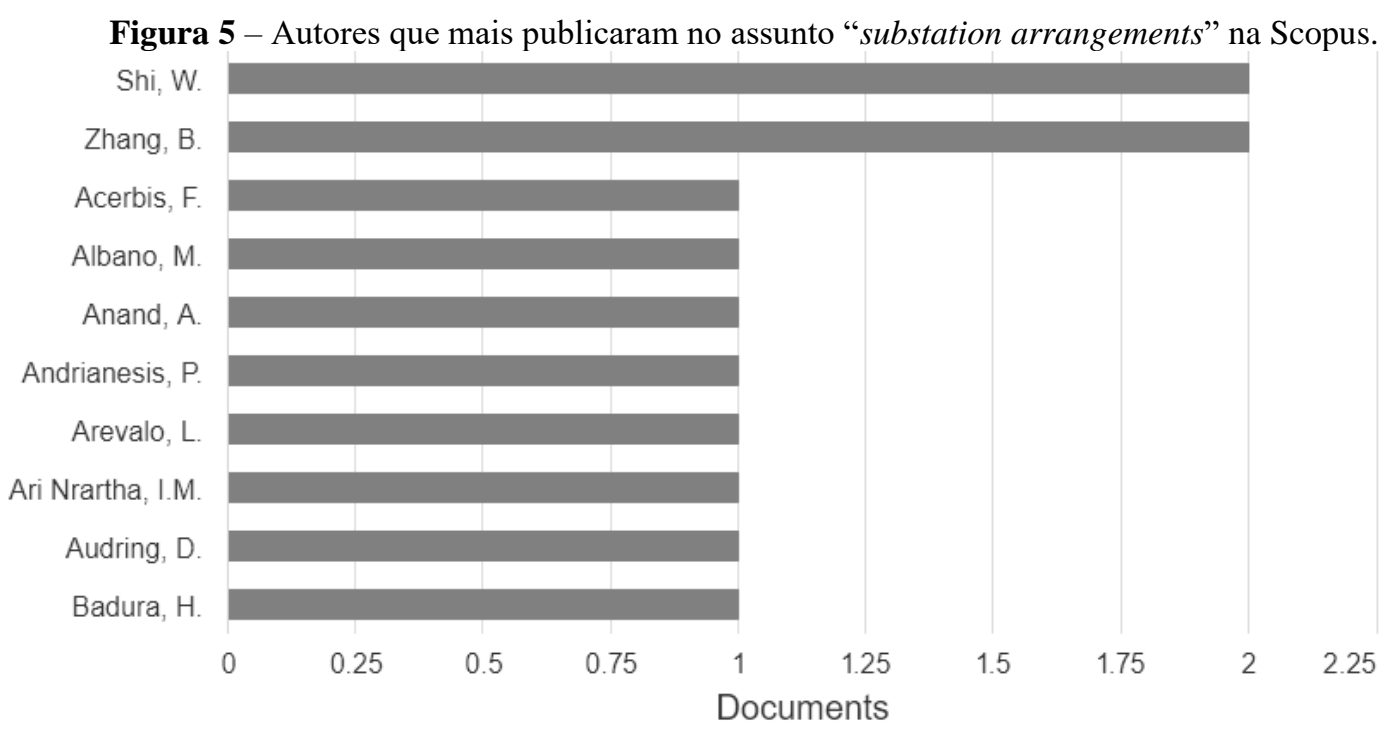

Fonte: Autores (2021).

Conforme disposto na Figura 6 não houve um grande volume de publicações nos últimos 5 anos relacionadas às palavras chaves "substation arrangements" na plataforma Scopus. Porém, quando se utiliza outras palavras chaves, a pesquisa retorna um número de produções acadêmicas maior. A seguir na Figura 7, a quantidade anual de publicação no assunto "substation arrangements":

Figura 6 - Quantidade de publicações no tema "substation arrangements" na Scopus.

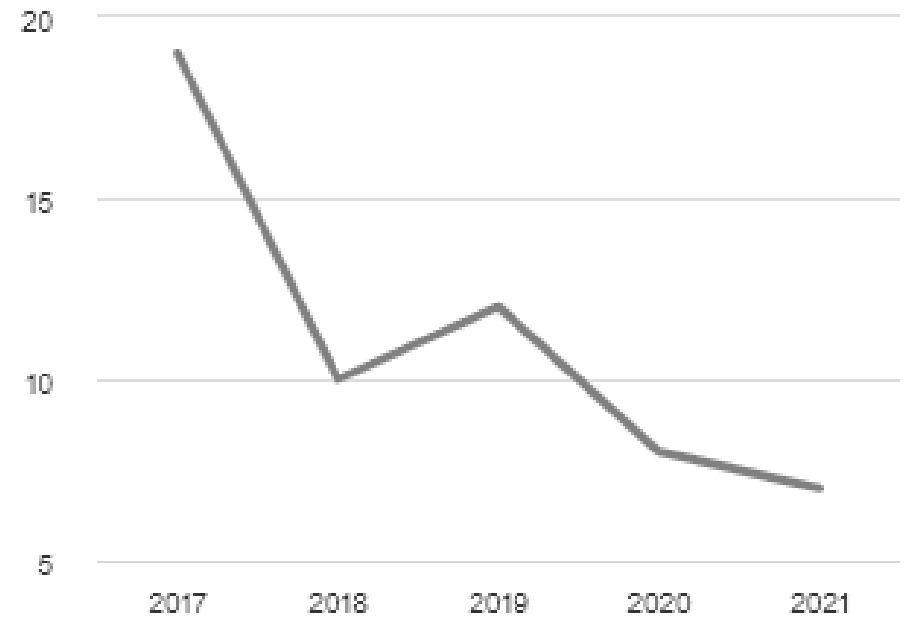

Fonte: Autores (2021).

Conforme indicado na Figura 7, o número de produções acadêmicas relacionadas ao tema de arranjo de subestações, utilizando na pesquisa as palavras chaves "substation arrangements" na plataforma Scopus demonstra que o ano de 2017 foi que mais houve publicações na temática nos últimos 5 anos e houve uma redução desde então.

A Figura 8 indica os países que mais publicaram documentos no período de 5 anos no assunto "substation arrangements": 
Figura 7 - Quantidade de publicações no tema "substation arrangements" por país na Scopus.

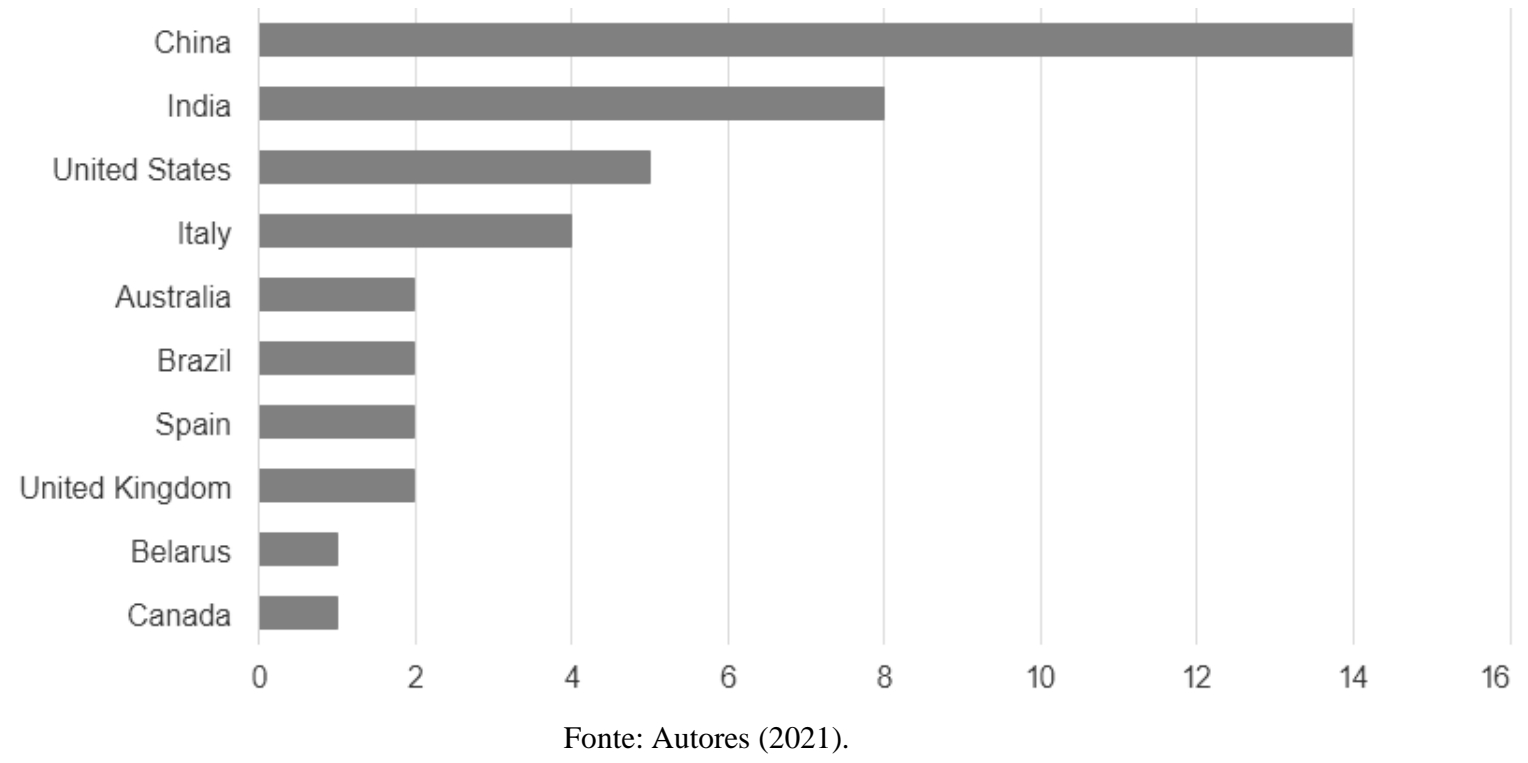

Em relação aos países que mais publicaram documentos no período de 5 anos no assunto "substation arrangements", destaque-se a China com 14 documentos relacionados. O Brasil aparece em sexto lugar conforme indicado na Figura 7.

No que concerne aos autores que mais publicaram nos últimos anos em relação às palavras chaves "substation arrangements", conforme levantamento bibliométrico realizado na plataforma Scopus, tem-se como principais referências os autores chineses Weidong Shi e Zhang B.

Em relação às publicações sobre extra alta tensão, recentemente, Weidong Shi publicou na $16^{\mathrm{a}}$ Conferência Internacional IET sobre Transmissão de Energia AC e DC (ACDC 2020) uma pesquisa sobre proteção contra raios em subestações de extra alta tensão $(500 \mathrm{kv})$ em redes elétricas no interior da Mongólia, país que faz fronteira com a China. O estudo é justificado devido à importância de uma proteção eficaz contra raios em uma subestação de extra alta tensão para que a rede opere de forma segura e estável.

\section{Conclusão}

Este artigo apresentou uma revisão bibliográfica sobre arranjo de subestações realizada através de uma análise bibliométrica sobre o tema. O estudo concentrou sua pesquisa nas plataformas Periódicos CAPES e Scopus e permitiu a análise de produções científicas publicadas direcionadas ao tema deste trabalho.

A análise bibliométrica nas duas plataformas, possibilitou obter-se um panorama sobre os estudos e pesquisas realizadas sobre subestações. Foi possível identificar nas duas plataformas pesquisadas que a publicação de artigos teve o maior volume em comparação com os demais tipos de publicação. Na plataforma CAPES encontrou-se apenas 3 artigos com a palavra chave arranjo de subestações, enquanto na plataforma Scopus foram encontradas 56 publicações.

Em um panorama mundial destaca-se a China com o maior número de produções científicas na área. O Brasil está no sexto lugar em produções relacionadas ao arranjo de subestações nos últimos cinco anos. Por fim, para continuidade desta pesquisa, sugere-se para trabalhos futuros um estudo aprofundado sobre novas tecnologias aplicadas e arranjo de modernas SEs no segmento da transmissão, distribuição e industrial. 
Research, Society and Development, v. 10, n. 14, e287101421805, 2021

(CC BY 4.0) | ISSN 2525-3409 | DOI: http://dx.doi.org/10.33448/rsd-v10i14.21805

\section{Referências}

Anderson, P. (1999) - Power Systems Protection. IEEE Press - Power Engineering.

Beeman, D., \& Beeman, D. (1955). Industrial power systems handbook (Vol. 195). McGraw-Hill.

Brandao, A. F. (2008). Reliability Reduction in Electrical Installations Due to Equipment Overload. IEEE Latin America Transactions, 6(1), 74-80.

Cabeza, L. F., Chàfer, M., \& Mata, É. (2020). Comparative analysis of web of science and scopus on the energy efficiency and climate impact of buildings.

Energies, 13(2), 409.

Colussi, A. (2002). Sistema especialista para avaliação de alternativas de topologia para subestações de transmissão.

Conceição, R. G., de Andrade, J. A. B., Ferreira, C. H., \& Alburqueque, C. J. (2016). Transmissão de energia elétrica em ultra alta tensão: o Complexo

Hidrelétrico Belo Monte. Revista Eletrônica Teccen, 9(1), 66-74.

de Oliveira, A., Fernandes, G., dos Anjos, R., Silva, T., \& Oliveira, E. (2017). Isolamento elétrico de subestações: Evolução da tecnologia e tendências. In 12th Latin-American Congress on Electricity Generation and Transmissiton (CLAGTEE 2017) (pp. 1-6).

Empresa de Pesquisa Energética (2020). Balanço Energético Nacional 2020: Ano base 2019. https://www.epe.gov.br/pt/publicacoes-

dadosabertos/publicacoes/balanco-energetico-nacional-2020.

Ferreira, N. S. D. A. (2002). As pesquisas denominadas "estado da arte". Educação \& sociedade, 23(79), $257-272$.

Giles, R. L. (1970). Layout of E. H. V. Substations. London: Cambridge University Press.

Heldt, V. (2016). Análise de atividades de montagem em subestações de transmissão de energia elétrica de alta tensão.

Leite, G. D. N. P., Weschenfelder, F., Araújo, A. M., Ochoa, Á. A. V., Neto, N. D. F. P., \& Kraj, A. (2019). An economic analysis of the integration between air-conditioning and solar photovoltaic systems. Energy Conversion and Management, 185, 836-849.

Liu, Z., Shi, W., \& Zhang, B. (2021). Numerical Analysis of Transient Performance of Grounding Grid with Lightning Rod Installed on Multi-Grounded Frame. Energies, 14(12), 3392.

Lu, T., Chen, X., Lei, T., Shen, H., Shi, W., Zhang, L., \& Miao, L. (2020, July). Research on lightning protection of $500 \mathrm{kV}$ extra-high voltage substation in west inner mongolia power grid. In The 16th IET International Conference on AC and DC Power Transmission (ACDC 2020), 1587-1592. IET.

Mamede Filho, J. (2019). Manual de Equipamentos de Alta tensão. LTC.

Mamede Filho, J., \& Mamede, D. R. (2013). Proteção de Sistemas Elétricos de Potência. LTC.

Marson, J.P. (2017). Metodologia de análise de arranjo de barras em subestações de alta tensão.

Operador Nacional do Sistema Elétrico (2020). Submódulo 2.3: Requisitos mínimos para subestações e seus equipamentos. http://www.ons.org.br/\%2FP roce di men tosDeRede\%2FM\%C3\%B3dulo\%202\%2FSubm\%C3\%B3dulo\%202.3\%2FSubm\%C3\%B3dulo\%202.3.pdf

Ruback, R. O., \& da Costa, V. M. (2018). A new method for analyzing three-phase faults under data uncertainties. IEEE Latin America Transactions, 16(5), 1395-1401.

Silveira, P. O. D. (2017). Planejamento de sistemas de transmissão em área com fonte de geração intermitente, apoiado no uso de tecnologias avançadas (Doctoral dissertation, Universidade de São Paulo).

Souza, F. R. D. (2003). Análise comparativa de arranjos de subestações por estudos de confiabilidade (Doctoral dissertation, Universidade de São Paulo). 